\title{
Distribution of candidemia in Malaysian tertiary care hospital revealed predominance of Candida parapsilosis
}

\author{
Yamin, D. ${ }^{*}$, Husin, A. ${ }^{2,3}$ and Harun, A. ${ }^{1,3}$ \\ ${ }^{1}$ Department of Medical Microbiology and Parasitology, School of Medical Sciences, Universiti Sains Malaysia, \\ 16150 Kubang Kerian, Kelantan, Malaysia \\ ${ }^{2}$ Department of Medicine, School of Medical Sciences, Universiti Sains Malaysia, 16150 Kubang Kerian, \\ Kelantan, Malaysia \\ ${ }^{3}$ Hospital Universiti Sains Malaysia, Jalan Raja Perempuan Zainab 2, 16150 Kota Bharu, Kelantan, Malaysia \\ *Corresponding author e-mail: dinayamin@student.usm.my \\ Received 13 January 2020; received in revised form 17 August 2020; accepted 18 August 2020
}

\begin{abstract}
Candida parapsilosis is an important pathogen of healthcare-associated bloodstream infections (BSI) causing high mortality and morbidity in immunocompromised patients in addition to other Candida species including C. albicans, C. tropicalis, C. glabrata, and $C$. krusei. Knowledge on recent local species distribution and trend is essential. An increase in the proportion of C. parapsilosis candidemia has been recently observed as a result of many risk factors. The distribution of candidemia has been changing in the last three decades. To determine the proportion of different Candida species causing candidemia in a tertiary-care hospital during January 2001 - December 2018, a retrospective study performed in a 853-bedded tertiary-care hospital in north-eastern Malaysia. All cases of candidemia from January-2001 to December-2018 were included, and the review was performed based on patients' medical records and laboratory database. The frequency of different Candida species was determined. This study showed that out of 1175 patients with candidemia, C. parapsilosis was the most common species contributing to $29.2 \%$ (343/1175) of candidemia, followed by C. albicans 20.1\% (236/1175), C. tropicalis 18.7\% (220/1175), C. glabrata 6.0\% (71/1175), C. guilliermondii $3.7 \%$ (43/1175), C. rugosa 1.9\% (22/1175), C. famata 1.7\% (20/1175), C. krusei $1.4 \%$ (16/1175), C. dubliniensis $0.8 \%$ (9/1175), C. lusitaniae $0.7 \%(8 / 1175)$, C. lipolytica $0.3 \%$ (4/1175), C. pelliculosa 0.3\% (4/1175), C. haemulonii, C. kefyr, C. utilis and C. inconspicua (1/1175 each). In addition, $14.9 \%$ (175/1175) belonged to Candida spp. which were not identified to species level. In conclusion, a different scenario for the proportion of Candida species with $C$. parapsilosis predominates over $C$. albicans as a nosocomial pathogen leading to candidemia has been shown in this study.
\end{abstract}

\section{INTRODUCTION}

The incidence of invasive Candida infections continues to rise worldwide, especially Candida bloodstream infections, due to many factors including increased use of immunosuppressive therapy and invasive devices (Giri \& Kindo, 2012; Mohamed et al., 2018).

In the USA, Candida has been reported as the fourth most common pathogen causing healthcare-associated bloodstream infections (BSIs) contributing $8-10 \%$ of the cases (Chi et al., 2011; Giri \& Kindo, 2012; Yapar, 2014; Amalina, 2018) and ranked sixth in Europe (Caggiano et al., 2015). Bloodstream infections due to Candida cause significant mortality and morbidity worldwide (Giri \& Kindo, 2012; Santhanam et al., 2013; Mohamed et al., 2018), especially in intensive care units (ICUs) and among immunocompromised patients (Noor, 2008; Tan et al., 2016). Knowledge of recent local trends, species distribution and antifungal resistance is essential for prevention and control strategies (Mohamed et al., 2018). 
Currently, Candida has been known to have more than 200 known species. However only a small proportion are of medical importance and have been isolated in human infections. These include Candida albicans, Candida glabrata, Candida tropicalis, Candida parapsilosis, Candida krusei, Candida guilliermondii, Candida lusitaniae and Candida dubliniensis. Of these species, $95 \%$ of the infections involved C. albicans, C. glabrata, C. parapsilosis, C. tropicalis, and C. krusei (Giri \& Kindo, 2012; Yapar, 2014). Recently a multidrug resistant species, Candida auris, has emerged as a health-care associated pathogen associated with mortality in hospitalized patients (Chowdhary et al., 2017).

Although C. albicans remains the major cause of invasive candidiasis in some regions, infections caused by non-albicans species have been recently observed with increasing trends worldwide, including Malaysia (Chi et al., 2011; Santhanam et al., 2013; Amalina, 2018; Mohamed et al., 2018). Among non-albicans species, C. parapsilosis was reported as one of the major pathogenic Candida in Latin America, Canada, Europe and Asia Pacific (Liu et al., 2018)

In particular, the incidence of infections by antifungal resistant non-albicans Candida has been increasing during the last several years. An important shift in the type of Candida infections, from C. albicans, towards a more treatment-resistant non-albicans Candida varieties has been observed. Therefore, epidemiological monitoring of pathogenic Candida is unquestionably important. Thus, the knowledge of local epidemiological trend of invasive candidiasis, mainly BSIs is an important guidance in therapeutic management (Noor, 2008; Chi et al., 2011; Kazak et al., 2014; Tan et al., 2016; Amalina, 2018).

Herein we report distribution of Candida species as causative agent for BSI at a tertiary hospital in Malaysia throughout 2001 and 2018.

\section{MATERIALS AND METHODS}

\section{Study Design}

This is a cross-sectional retrospective study conducted to analyze episodes of candidemia among patients at Hospital Universiti Sains Malaysia (USM), Malaysia from 1st January 2001 to 31st December 2018. Hospital USM is a 853-bedded tertiary government hospital, with intensive care units and oncology wards. This study was approved by the Human Research Ethics of Universiti Sains Malaysia [USM/JEPeM/ 16040162].

\section{Clinical and Laboratory Data}

Data regarding Candida isolation from blood samples and patients' clinical information were obtained from the Hospital USM Laboratory Information System and Medical Records Unit.

\section{Definitions}

Candidemia was defined as at least one positive blood culture for Candida species in patients with symptoms or signs of infection, whether it was isolated from peripheral or central line.

Candida spp. refers to non-albicans Candida species that were not identified to species level.

\section{Case Selection}

Inclusion criteria: All in-patients with candidemia from January-2001 to December2018 were included. Candidemia that occurred more than 30 days after the initial episode were considered as a new episode. Candidemia episodes in same patient but by different species were considered as separate episodes.

Exclusion criteria: Repetitive blood cultures obtained from the same patient with the same Candida species within one month were excluded. 


\section{Candida Isolation and Identification}

The isolation of Candida has been performed routinely using standard mycology methods. Commercially available identification systems, namely API 20C AUX® or ID 32C® biochemical identification kit (BioMérieux, Marcy I'Etoile, France) or VITEK® system (BioMérieux, Marcy I'Etoile, France) (last two years) have been used for identification of Candida to species level. For API 20C AUX ${ }^{\circledR}$ and ID 32C®, identification percentage threshold of more than $88 \%$ was considered as acceptable. Molecular identification using ribosomal RNA gene sequencing was also performed to confirm some isolates with difficult phenotypic identification.

\section{Statistical Analysis}

Data was analyzed using SPSS software for windows (Version 24.0; SPSS, Chicago, IL, USA). Descriptive statistical analysis was done. The distribution of different Candida species was expressed as proportion and relative frequency.

\section{RESULTS}

\section{Species Distribution}

A total of 1175 non-repetitive cases of candidemia were identified from January 2001 to December 2018 (Table 1). C. parapsilosis was the most commonly isolated species contributing to $29.2 \%$ (343/1175) of candidemia, followed by C. albicans $20.1 \%$
(236/1175). Our data shows that three quarters of all candidemia were due to the four main Candida species, C. parapsilosis, C. albicans, C. tropicalis, and C. glabrata, and their proportions were $29.2 \%, 20.1 \%$, $18.7 \%$ and $6.0 \%$ respectively. Other isolated Candida species include C. guilliermondii 3.7\% (43/1175), C. rugosa 1.9\% (22/1175), C. famata $1.7 \%$ (20/1175), C. krusei $1.4 \%$ (16/1175), C. dubliniensis 0.8\% (9/1175), C. lusitaniae $0.7 \%$ (8/1175), C. lipolytica $0.3 \%$ (4/1175), C. pelliculosa $0.3 \%$ (4/1175). The rarer ones, C. haemulonii, C. kefyr, C. utilis and $C$. inconspicua contributed one isolate each $(1 / 1175)$.

The trend of the three most common Candida species has been changing over the period of the study (Figure 1). In 2001 the relative frequency of $C$. albicans was the highest, followed by $C$. parapsilosis which then predominated over other Candida species, and reached its peak in 2006, in which it accounted for $38 \%$ of all candidemia. In 2012 candidemia cases were almost equally distributed between the three Candida species. In the last six years C. parapsilosis continued to outnumber both $C$. albicans and $C$. tropicalis except in 2018, where $C$. tropicalis outnumbered C. parapsilosis and consequently C. albicans, accounting to $47 \%$ of all candidemia.

A total of 622 C. parapsilosis blood isolates have been isolated from patients admitted to different wards from 2001 to 2018 (Figure 2). Out of 622 C. parapsilosis

Table 1. Distribution of Candida species at HUSM from January 2001 to December 2018

\begin{tabular}{lcc}
\hline Organism & Number of patients & Percentage \% \\
\hline Candida parapsilosis & 343 & 29.2 \\
Candida albicans & 236 & 20.1 \\
Candida tropicalis & 220 & 18.7 \\
Candida spp. & 175 & 14.9 \\
Candida glabrata & 71 & 6.0 \\
Candida guilliermondii & 43 & 3.7 \\
Candida rugosa & 22 & 1.9 \\
Candida famata & 20 & 1.7 \\
Candida krusei & 16 & 1.4 \\
Candida dubliniensis & 9 & 0.8 \\
Candida lusitaniae & 8 & 0.7 \\
Others & 12 & 1.0 \\
\hline
\end{tabular}




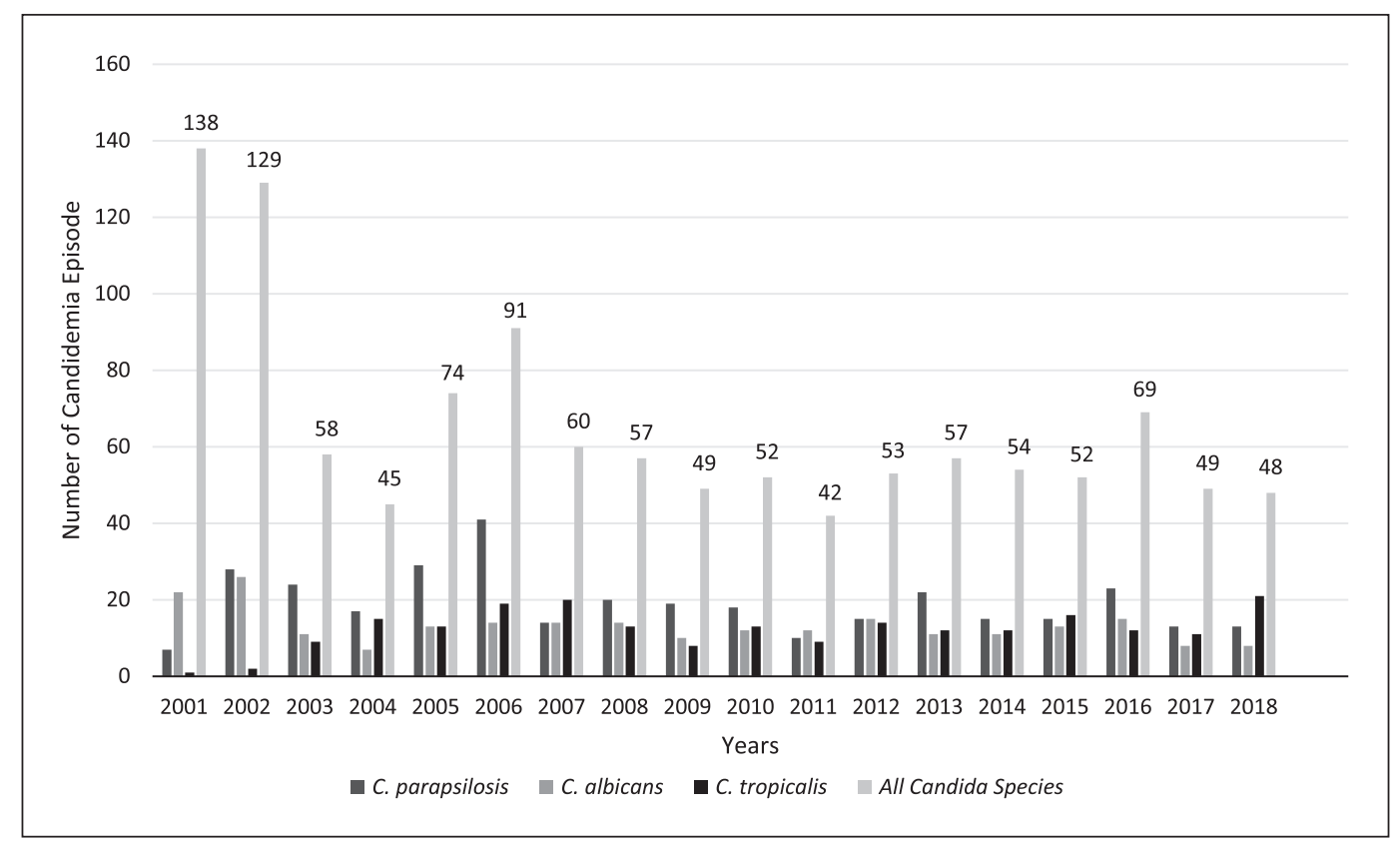

Figure 1. Trends of main Candida species from 2001 to 2018.

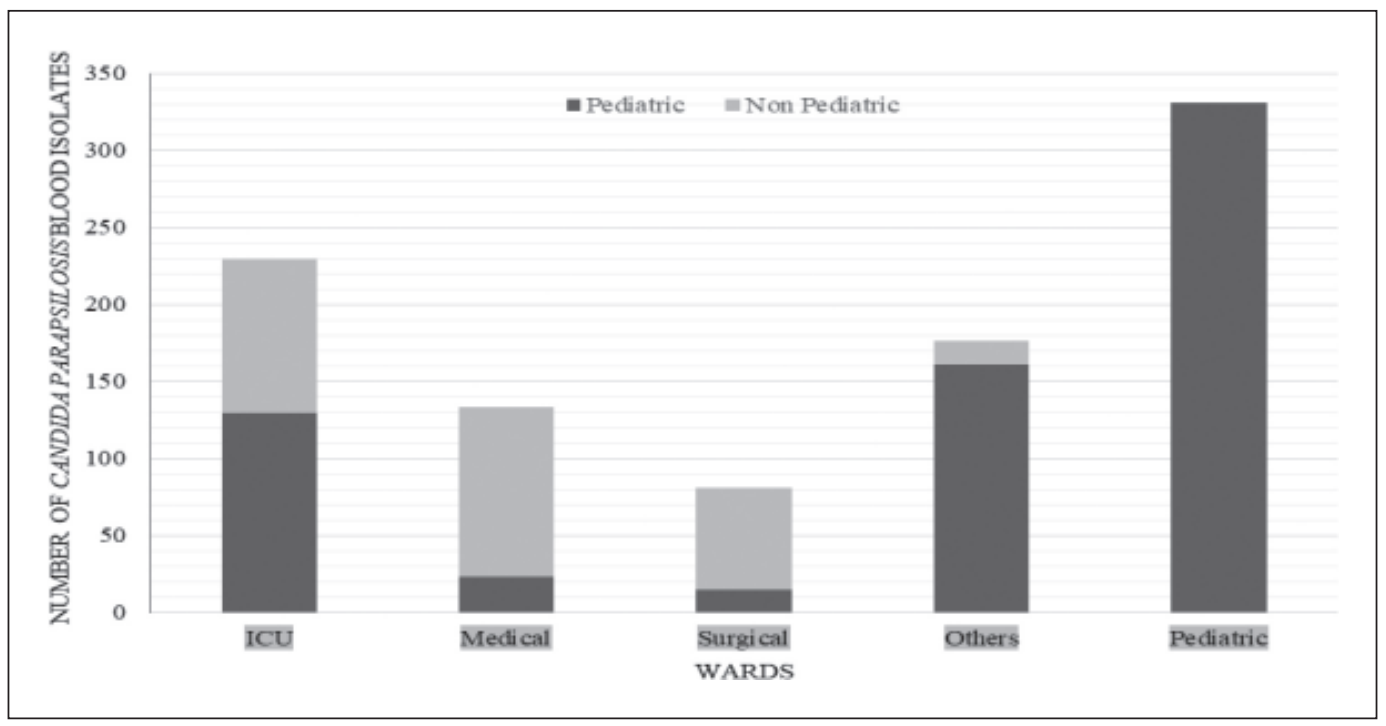

Figure 2. Distribution of C. parapsilosis in different wards (2001-2018).

positive blood cultures, 229 (36.8\%) isolates were isolated from ICU patients, 133 (21.4\%) from medical wards, 81 (13.0\%) from surgical wards, and 179 (28.8\%) from other locations. Collectively, 331 out of the 622 C. parapsilosis isolates were from pediatric wards.

\section{DISCUSSION}

Candida species are the most common cause of invasive yeast infections. At least 16 recognized species of Candida cause human diseases, while more than $90 \%$ of infections are caused by the five most 
common pathogens, C. albicans, C. glabrata, C. tropicalis, C. parapsilosis, and C. krusei (Mohamed et al., 2018). Many factors contribute to the occurrence of candidiasis, including advances in medicine, expansion of human life span and immunodeficiencies. Advances in medicine such as transplantation of organs and tissues, application of total parenteral feeding and parenteral administration of antimicrobial therapies do not only expand human life but also increase the number of susceptible patients to opportunistic infections. Immunodeficiency including the widely spread human immunodeficiency virus (HIV) infection and long-term peroral antibiotics administration enhance the susceptibility to systemic fungal infection, which is a great problem, due to its diagnostic difficulties. Therefore, prevention of systemic infections is extremely important, and accomplished by removal of predisposing factors as well as well-controlled objective drug administration (Noor, 2008).

This study provides data on species distribution of Candida isolated from patients' blood samples at a tertiary hospital over 18 years period, in which non-albicans Candida accounted for $79.9 \%$ of the total blood Candida isolates obtained throughout year 2001 to 2018 , from which 160 out of 175 candidemia cases were not identified to species level during 2001-2002 until the introduction of API 20C AUX® kit for species identification. Our study has shown different scenario in the proportion of Candida species whereby C. parapsilosis predominates over C. albicans, in contrast to previous studies conducted in Malaysia and neighboring countries, where $C$. albicans was the major cause of candidemia (Tan et al., 2016; Mohamed et al., 2018).

In this study, C. parapsilosis was found to be the most common yeast isolated from blood specimens. The organism outnumbered C. albicans by $9.1 \%$ throughout 18 years, a trend not observed before in other Malaysian studies. Our finding also demonstrated the important role of nonalbican Candida particularly C. parapsilosis as a cause of bloodstream infections in our health care setting. C. parapsilosis was the most common species isolated from blood accounting for $29.2 \%$ of isolates, followed by $C$. albicans and C. tropicalis. The largest proportion of $C$. parapsilosis isolation was from intensive care units (both adults and pediatrics), while the smallest proportion were from surgical wards. These findings are in line with other published studies, where C. parapsilosis accounted for significantly more cases than $C$. albicans and C. tropicalis (Noor, 2008; Amalina, 2018). Similarly, the results of a population-based surveillance study in Barcelona, Spain indicated that $C$. parapsilosis isolates constituted $23 \%$ of all studied fungemia (Almirante et al., 2006).

On the other hand, other studies have reported different findings, in which C. tropicalis was the most common Candida species isolated from blood (Tzar \& Shamim, 2009; Ng et al., 2015; Mohamed et al., 2018). Santhanam et al. (2013) has reported $C$. albicans as the predominant species causing candidemia (33.9\% of bloodstream isolates) in their setting (Santhanam et al., 2013). In another study involving a total of 1062 Candida isolates, C. albicans accounted for $43.8 \%(\mathrm{n}=465)$, followed by C. parapsilosis (26.5\%), C. tropicalis (8.4\%), C. krusei (5.8\%), and C. glabrata (5.5\%) (Kazak et al., 2014). In a 16-year-study from Italy, C. albicans was reported to be the predominant species (44.2\%) (Caggiano et al., 2015). In a study from Taiwan, C. glabrata was shown to be the most frequently isolated non-albicans Candida species (Chi et al., 2011). A multicenter study reported $C$. albicans as the predominant species, but its proportion was lesser than non-albicans Candida species collectively (Tan et al., 2016).

The relative frequency of non-albicans Candida among Candida species in hospitalbased studies done in various parts of the world seem to largely differ from $18.3 \%$ to $83.0 \%$. This variability between various geographical regions is multifactorial, mostly due to different antifungal therapies and treatment practices, frequency of using invasive procedures such as use of indwelling catheters, patient demography and chronic underlying illnesses (Mohamed et al., 2018). 
Furthermore, species vary geographically in frequency of isolation, often because of different patient populations that may reflect differences in healthcare practices among different countries in the populations studied as well as differences in study methodologies adopted by different authors, or the systems for regular surveillance (Yapar, 2014; Caggiano et al., 2015). This variability in the most frequent cause of candidemia has been explained partly by the high affinity of C. parapsilosis to intravascular devices and parenteral nutrition that were used widely in recent years (Almirante et al., 2006).

The high profusion of $C$. parapsilosis could be clarified by factors related to hospital settings, such as gastrointestinal colonization, or the adhesion to intravascular devices and prosthetic materials. Additionally, C. parapsilosis has been known to be isolated from the hands of healthcare workers and is often responsible for nosocomial clusters, so its presence in hospital settings may be explained by insufficient implementation of infection control practices (Caggiano et al., 2015). C. parapsilosis commonly colonize skin surface and has a better affinity to materials like acrylic in glucose-containing solutions and TPN solutions. It is obviously known to cause bloodstream infections in infants and neonates. The detection of $C$. parapsilosis in bloodstream infections indicates exogenous introduction of the pathogen from the surrounding environment (Giri \& Kindo, 2012).

As has been expected in previous studies, non-albicans Candida continue to emerge as an important cause of serious disease in our health care setting by (Noor, 2008). Therefore, ongoing surveillance of serious infections caused by Candida species is important to track changes in the epidemiology and consequently in antifungal susceptibility among these emerging pathogens. In the present study, C. parapsilosis blood isolates were widely distributed across multiple hospital wards, in which intensive care units were the major sources accounting for $37 \%$ of the isolates collectively. Our results were in concordance with a previous study where C. parapsilosis were frequently isolated from intensive care settings and associated with high mortality rate (Liu et al., 2018). A review of antifungal susceptibility profiles of $C$. parapsilosis in our setting revealed $94.4 \%$ of susceptibility to amphotericin B, 84.8\% to fluconazole, $98.8 \%$ to voriconazole and $88.2 \%$ to caspofungin (data not shown). Thus, the results of this study support fluconazole as favorable antifungal drug for empirical and pre-emptive treatment of $C$. parapsilosis infections in our setting. Echinocandins has been recommended in azole-resistant Candida infections, whereas lipid formulation amphotericin $\mathrm{B}$ is recommended for patients with suspected azole- and echinocandinresistant Candida infections (Tissot et al., 2017; Bassetti et al., 2018). Although the susceptibility of $C$. parapsilosis to currently available antifungal agents is generally predictable, antifungal susceptibility testing is strongly recommended because individual isolates of same species may not necessarily follow this general pattern.

\section{CONCLUSION}

In this 18-year study on Candida species blood isolates, a shift towards isolation of non-albicans Candida has been observed. Recently C. parapsilosis was noted to be the most predominant species. Continuous surveillance on the species prevalence and antifungal susceptibility profiles are needed to guide the clinicians in empirical and preemptive treatment of invasive candidiasis. Accurate epidemiology of invasive Candida infections is important to support institutional, national, and regional guidelines for the best management of invasive fungal infections.

Acknowledgement. We would like to acknowledge the Department of Medical Microbiology and Parasitology, for the access to laboratory and clinical data. This study was supported by Universiti Sains Malaysia RUI grant 100/PPSP/812206 awarded to Azian Harun. 


\section{Conflict of Interests}

The authors declare that they have no conflict of interests.

\section{REFERENCES}

Almirante, B., Rodríguez, D., Cuenca-Estrella, M., Almela, M., Sanchez, F., Ayats, J., Alonso-Tarres, C., Rodriguez-Tudela, J.L., Pahissa, A. \& Group, B.C.P.S. (2006). Epidemiology, risk factors, and prognosis of Candida parapsilosis bloodstream infections: case-control population-based surveillance study of patients in Barcelona, Spain, from 2002 to 2003. Journal of Clinicl Microbiology 44(5): 1681-1685.

Amalina, H. (2018). Epidemiology and Outcomes of Candidaemia among Adult Patients Admitted at Hospital Universiti Sains Malaysia (HUSM): A 5-Year Review. Epidemiology 17(1).

Bassetti, M., Righi, E., Montravers, P. \& Cornely, O.A. (2018). What has changed in the treatment of invasive candidiasis? A look at the past 10 years and ahead. Journal of Antimicrobial Chemotherapy 73(Suppl_1): i14-i25.

Caggiano, G., Coretti, C., Bartolomeo, N., Lovero, G., De Giglio, O. \& Montagna, M.T. (2015). Candida Bloodstream Infections in Italy: Changing Epidemiology during 16 Years of Surveillance. BioMed Research Intermational 2015: 256580.

Chi, H.-W., Yang, Y.-S., Shang, S.-T., Chen, K.-H., Yeh, K.-M., Chang, F.-Y. \& Lin, J.-C. (2011). Candida albicans versus nonalbicans bloodstream infections: the comparison of risk factors and outcome. Journal of Microbiology, Immunology and Infection 44(5): 369-375.

Chowdhary, A., Sharma, C. \& Meis, J.F. (2017). Candida auris: A rapidly emerging cause of hospital-acquired multidrugresistant fungal infections globally. PLoS Pathogens 13(5): e1006290.

Giri, S. \& Kindo, A.J. (2012). A review of Candida species causing blood stream infection. Indian Journal of Medical Microbiology 30(3): 270-278.
Kazak, E., Akın, H., Ener, B., Sığırlı, D. Özkan, Ö., Gürcüoğlu, E., Yılmaz, E., Çelebi, S., Akçağlar, S. \& Akalın, H. (2014). An investigation of Candida species isolated from blood cultures during 17 years in a university hospital. Mycoses 57(10): 623-629.

Liu, Y., Kang, M., Ye, H., Zong, Z. \& Lv, X. (2018). Analysis on clinical characteristics and drug resistance of Candida parapsilosis bloodstream infections in West China Hospital, China, from 2012 to 2015. Journal de Mycologie Medicale 28(1): 222-226.

Mohamed, N.A., Pathmanathan, S.G., Hussin, H. \& Zaini, A.B. (2018). Distribution and antifungal susceptibility pattern of Candida species at a Tertiary hospital in Malaysia. The Journal of Infection in Developing Countries 12(02): 102-108.

Ng, K.P., Kuan, C.S., Kaur, H., Na, S.L., Atiya, N. \& Velayuthan, R.D. (2015). Candida species epidemiology 2000-2013: a laboratory-based report. Tropical Medicine \& International Health 20(11): 1447-1453.

Noor, S.S.M. (2008). Epidemiology of Candida species in tertiary-teaching hospital in Malaysia. Intermational Medical Journal 15(4): 291-294.

Santhanam, J., Nazmiah, N. \& Aziz, M.N. (2013). Species distribution and antifungal susceptibility patterns of Candida species: is low susceptibility to itraconazole a trend in Malaysia? The Medical Journal of Malaysia 68(4): 343-347.

Tan, T.Y., Hsu, L.Y., Alejandria, M.M. Chaiwarith, R., Chinniah, T., Chayakulkeeree, M., Choudhury, S., Chen, Y.H., Shin, J.H., Kiratisin, P., Mendoza, M., Prabhu, K., Supparatpinyo, K., Tan, A.L., Phan, X.T., Tran, T.T., Nguyen, G.B. Doan, M.P., Huynh, V.A., Nguyen, S.M., Tran, T.B. \& Van Pham, H. (2016). Antifungal susceptibility of invasive Candida bloodstream isolates from the Asia-Pacific region. Medical Mycology 54(5): 471-477. 
Tissot, F., Agrawal, S., Pagano, L., Petrikkos, G., Groll, A.H., Skiada, A., Lass-Florl, C., Calandra, T., Viscoli, C. \& Herbrecht, R. (2017). ECIL-6 guidelines for the treatment of invasive candidiasis, aspergillosis and mucormycosis in leukemia and hematopoietic stem cell transplant patients. Haematologica 102(3): 433-444.
Tzar, M. \& Shamim, A. (2009). Candidaemia and antifungal susceptibility testing in a teaching hospital. The Medical Journal of Malaysia 64(1): 61-64.

Yapar, N. (2014). Epidemiology and risk factors for invasive candidiasis. Therapeutics and Clinical Risk Management 10: 95-105. 